\title{
Vielzahl neuer therapeutischer Möglichkeiten
}

\author{
Neue Ursachenforschung, neue Diagnoseverfahren, neue Klassifikationen
}

„Innerhalb des großen Pools der Lymphome passiert viel“, stellte Univ.-Prof. Dr. Markus Raderer, Univ.-Klinik für Innere Medizin I der Medizinischen Universität Wien, zum Thema Kutane Lymphome fest. In der Gesellschaft der Ärzte in Wien versuchten Internisten, Onkologen und Dermatologen aus interdisziplinärer Sicht den gegenwärtigen Wissensstand über Cutane Lymphome und Lymphome mit sekundärer Hautbeteiligung darzustellen.

Eine Reihe von Erregern wurde in jüngster Zeit identifiziert, stellte Raderer fest: Epstein-Barr-Virus, HIV, Hepatitis-Viren, Borrelien, Chlamydien, und insbesondere Heliopacter pylori, die als einzige im Magen überleben können und eine Infektionsrate von etwa 50 Prozent aufweisen. Eine Impfung sei in Vorbereitung, wobei die vollständige Elimination des Heliobacter notwendig sei. Voraussetzung für eine erfolgreiche Therapie ist die Verifizierung der Ursache, hier sei jedoch noch viel Arbeit zu leisten. Bei Virus-verursachten Lymphome erziele man nur mit einer virusgezielten Therapie - etwa Interferon - einen Benefit, bei Chlamydien mit Doxycyclin.

\section{Klassifikation erleichtert Entscheidung}

Noch gibt es verschiedene Nomenklaturen für ein Krankheitsbild, das allerdings in vielfältigen Ausprägungen erscheint, konstatierte Univ.-Prof. Dr. Franz Trautinger, Landesklinik St. Pölten, Abteilung für Haut- und Geschlechtskrankheiten. WHO und EORTC streben eine Koordination der Klassifikation, des Staging und der Leitlinien an. Grundsätzlich muss in T-Zell und in B-Zell Lymphome unterschieden werden, wonach such auch die Therapie richtet. Besonders häufig sind T-Zell Lymphome, die sehr aggressiv sein können. So beträgt etwa die mittlere Überlebenszeit ab dem Zeitpunkt der histologischen Diagnose bei der erythrodermatischen Form der Mycosis fungoides ohne entsprechende Behandlung weniger als fünf Jahre. B-ZellLymphome treten oft in niedriger Malignität auf, wie das häufige Zentroblastisch-zentrozystische Lymphom. Von höherer Malignität ist das Lymphoblastische Lymphom.

Die Behandlungsstrategien müssen auf Grund einer exakten Diagnose erfolgen und die Vorbehandlung sowie das Tumorstadium berücksichtigen. Es liegen nur wenige kontrollierten Studien vor „und es muss auch nicht jeder rote Fleck völlig wegbehandelt werden", bemerkte Trautinger. Für die häufigeren T-Zell Lymphome wird eine stadiengerechte, eher zurückhaltende Therapie empfohlen. In frühen Stadien stehen topische Steroide, PUVA, lokal applizierte Zytostatika oder eine Radiotherapie im Vordergrund. Im fortgeschrittenen Stadium bieten sich systemische Therapien an, etwa PUVA in Kombination mit Retinoiden. Bei B-ZellLymphomen niedriger Malignität ist eine lokale Röntgenbestrahlung ausreichend, bei höher malignen Lymphomen ist zusätzlich eine Chemotherapie erforderlich.

\section{Lymphome mit sekundärer Hautbeteiligung}

Jene Varianten, die erst sekundär Symptome in der Haut hervorrufen, wie Leukämie, Lymphknotenkrebs, müssten möglichst früh erkannt werden, forderte Univ. Prof. Dr. Klaus Geissler, Onkologe und
Hämatologe im KH Hietzing. Die genaue Zuordnung zur jeweiligen Grundkrankheit bedeutet eine wichtige Entscheidungshilfe für die Auswahl der Therapie, die vom defensiven Abwarten bis zur Elimination der hämatopoetischen Zellen des Patienten mit einer anschließenden Stammzellimplantation reichen kann.

\section{Neue Therapieansätze}

„Neben der genauen Diagnose ist für die Therapieentscheidung das Tumorstadium zu berücksichtigen“, erklärte Univ. Prof. Dr. Beatrix Volc-Platzer Dermatologische Abteilung, Donauspital SMZ-Ost. Hierzu gebe es jedoch wenige kontrollierte Studien und wechselnde Klassifikationen. Sie empfiehlt eine gewisse Beobachtungszeit und rät von einem „overtreatment" bei niedriger Maglinität ab. In vielen Fällen, etwa beim Sezary-Syndrom, ist eine Kombinationstherapie indiziert: PUVA, Bexaroten und extrakorporale Photopherese, die einen fixen Platz in der Therapie hat. Andere Möglichkeiten betreffen die Vorantreibung der Apoptose durch das Fusionsprotein Denileukin diftitox und durch HistondeacetylaseInhibitoren. Ausschlaggebend ist der klinische Benefit. Die neuen Trends liegen bei Biologic response modifieres, Interferon, Retinoiden, Antikörpern, Knochenmarkstransplantationen und Tumorvakzinen.

Aus dieser Vielzahl der neuen therapeutischen Möglichkeiten muss für den Patienten eine individuelle Auswahl getroffen werden. Um dem stets wachsenden Angebot Rechnung zu tragen, werden von der Europäischen Kommission und der WHO jährlich entsprechende Leitlinien evaluiert.

Dr. Gerta Niebauer 